\title{
High-energy electronic interaction in the $3 d$ band of high-temperature iron-based superconductors
}

\author{
D. V. Evtushinsky, ${ }^{1, *}$ A. N. Yaresko, ${ }^{2}$ V. B. Zabolotnyy, ${ }^{1, \dagger}$ J. Maletz, ${ }^{1}$ T. K. Kim, ${ }^{3}$ A. A. Kordyuk, ${ }^{1,4}$ M. S. Viazovska, ${ }^{5, \ddagger}$ \\ M. Roslova, ${ }^{1,6}$ I. Morozov, ${ }^{1,6}$ R. Beck, ${ }^{1}$ S. Aswartham, ${ }^{1}$ L. Harnagea, ${ }^{1}$ S. Wurmehl, ${ }^{1,7}$ H. Berger, ${ }^{8}$ V. A. Rogalev, ${ }^{9, \dagger}$ \\ V. N. Strocov, ${ }^{9}$ T. Wolf, ${ }^{10}$ N. D. Zhigadlo, ${ }^{11,12}$ B. Büchner, ${ }^{1,7}$ and S. V. Borisenko ${ }^{1}$ \\ ${ }^{1}$ Institute for Solid State Research, IFW Dresden, P.O. Box 270116, D-01171 Dresden, Germany \\ ${ }^{2}$ Max-Planck-Institute for Solid State Research, Heisenbergstrasse 1, D-70569 Stuttgart, Germany \\ ${ }^{3}$ Diamond Light Source, Harwell Science and Innovation Campus, Didcot OX11 ODE, United Kingdom \\ ${ }^{4}$ Institute of Metal Physics of National Academy of Sciences of Ukraine, 03142 Kiev, Ukraine \\ ${ }^{5}$ Humboldt University of Berlin, Rudower Chaussee 25, D-12489 Berlin, Germany \\ ${ }^{6}$ Moscow State University, 119991 Moscow, Russia \\ ${ }^{7}$ Institut für Festkörperphysik, Technische Universität Dresden, D-01171 Dresden, Germany \\ ${ }^{8}$ Institut de Physique Applique, Ecole Politechnique Federale de Lausanne, CH-1015 Lausanne, Switzerland \\ ${ }^{9}$ Swiss Light Source, Paul Scherrer Institute, CH-5232 Villigen PSI, Switzerland \\ ${ }^{10}$ Karlsruher Institut für Technologie, Institut für Festkörperphysik, D-76021 Karlsruhe, Germany \\ ${ }^{11}$ Department of Chemistry and Biochemistry, University of Bern, CH-3012 Bern, Switzerland \\ ${ }^{12}$ Solid State Physics Laboratory, ETH Zurich, CH-8093 Zurich, Switzerland
}

(Received 7 April 2017; revised manuscript received 23 May 2017; published 1 August 2017)

\begin{abstract}
One of the most unique and robust experimental facts about iron-based superconductors is the renormalization of the electronic band dispersion by factor of 3 and more near the Fermi level. Obviously related to the electron pairing, this prominent deviation from the band theory lacks understanding. Experimentally studying the entire spectrum of the valence electrons in iron arsenides, we have found an unexpected depletion of the spectral weight in the middle of the iron-derived band, which is accompanied by a drastic increase of the scattering rate. At the same time, the measured arsenic-derived band exhibits very good agreement with theoretical calculations. We show that the low-energy Fermi velocity renormalization should be viewed as a part of the modification of the spectral function by a strong electronic interaction. Such an interaction with an energy scale of the whole $d$ band appears to be a hallmark of many families of unconventional superconductors.
\end{abstract}

DOI: 10.1103/PhysRevB.96.060501

There is a fundamental problem in condensed matter physics: The Hamiltonian of any solid is extremely complicated due to the large amount of involved particles. It is actually not trivial that one-electron mean-field theory (MFT) gives astonishingly accurate predictions for the electronic properties of a vast majority of common materials [1-3]. On the other hand, the one-electron approach spectacularly fails for many compounds that are currently under the scrutiny of modern condensed matter physics, especially for those with potentially useful extraordinary properties $[4,5]$. One recent example is the class of iron-based high-temperature superconductors [6,7], where numerous experimental techniques have established that the distribution of the electronic states at the Fermi level is compressed three and more times as compared to the MFT predictions [8-13]. Since there are many candidates not accounted for by the MFT approximation, which could lead to the renormalization at low energies, and very little is known experimentally about the nature of the force capable of triple band squeezing, the problem remains unsolved. In this Rapid Communication we apply angle-resolved photoemis-

\footnotetext{
*Present address: Institute of Physics, Ecole Polytechnique Federale de Lausanne (EPFL), CH-1015 Lausanne, Switzerland.

${ }^{\dagger}$ Present address: Physikalisches Institut und Röntgen Center for Complex Materials Systems (RCCM), Universität Würzburg, Am Hubland, 97074 Würzburg, Germany.

${ }^{\ddagger}$ Present address: Institute of Mathematics, Ecole Polytechnique Federale de Lausanne (EPFL), CH-1015 Lausanne, Switzerland.
}

sion spectroscopy (ARPES) to study the electronic structure of iron-based superconductors covering a much larger interval of binding energies than it is usually done, and show that not only electronic states at the Fermi level are renormalized, but the whole structure of the iron $3 d$ band is changed with respect to MFT by a strong interaction of an energy scale comparable to the total bandwidth.

In Fig. 1 we compare ARPES spectra recorded along the high-symmetry directions with the corresponding results of the local density approximation (LDA) band structure calculations for a NaFeAs compound. At the bottom of the valence band, between 2 and $5 \mathrm{eV}$ binding energies $(\omega)$, the photoemission intensity closely follows the calculated dispersions which correspond to the $p$ bands of arsenic. In this region there are well-defined dispersion features with moderate broadening and no appreciable renormalization. Near $\omega \approx 2 \mathrm{eV}$, where the bottom of the iron $3 d$ band is located, we observe the features with significantly larger scattering, but still a noticeable dispersion and energy position similar to the original nonrenormalized LDA bands. A comparison of the dispersions at even lower binding energies demonstrates that the experimental features change rapidly from smeared out and weakly defined to intense and well-discernible ones when going from $500 \mathrm{meV}$ to 0 . It is clearly seen that they are located much closer to the Fermi level than the calculations predict [compare Fig. 1(a) with Figs. 1(b) and 1(c) and Fig. 1(e) with Figs. 1(f) and 1(g), respectively]. In some spectra one is able to see a kink in the dispersion at about $500 \mathrm{meV}$ [Figs. 1(c), 1(g) and 1(h)]. See the Supplemental Material [14] 
(a) band dispersion

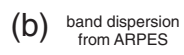

(c)

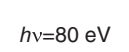

(d) $\quad h_{v=100 \mathrm{eV}}$

$(\mathrm{e}) \underset{\text { from calculation }}{\text { band dispersion }}(\mathrm{f}) \underset{\text { from ARPES }}{\text { band dispersion }}(\mathrm{g})$

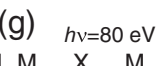

(h)

(h) $\quad h v=160 \mathrm{ev}$
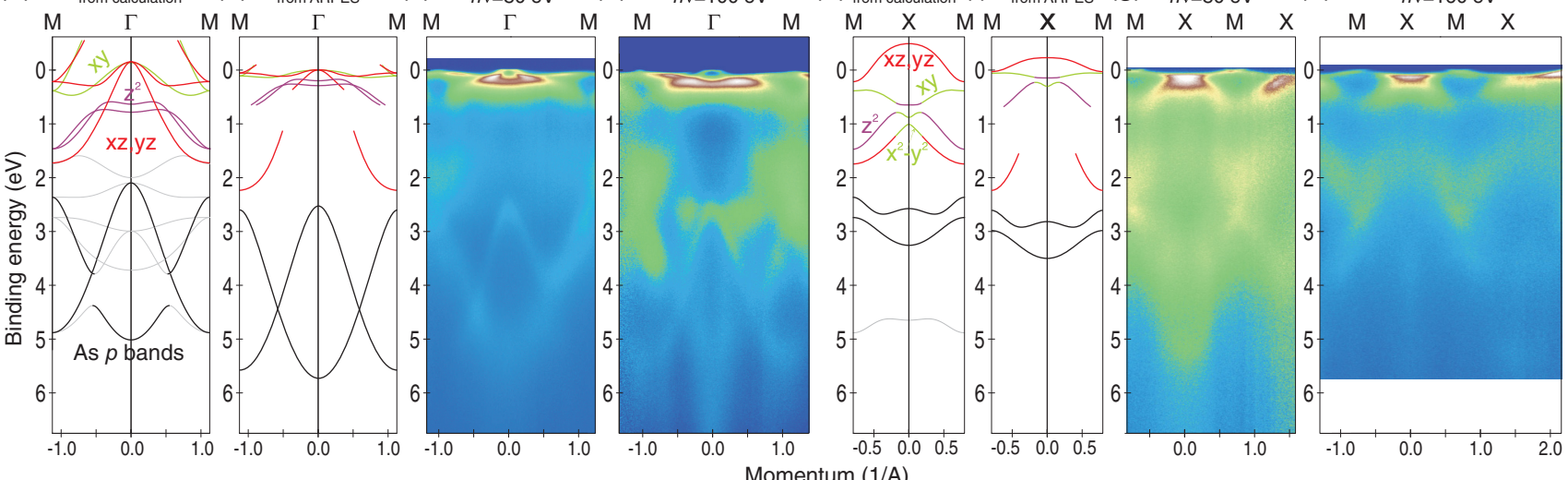

FIG. 1. Theoretically calculated and derived from ARPES band dispersion in NaFeAs. (a) Calculated band dispersion in the $M \Gamma M$ direction. Color coding of the electronic state orbital composition is indicated in the figure. Orbital polarization of the electronic states in this direction is very strong. (b) Contours of the band dispersion, extracted from an ARPES intensity distribution. Extraction of experimental dispersion curves relies on the data measured at different experimental conditions; typical spectra are shown in (c) and (d). (e)-(h) present the same information for the $M X M$ direction.

for a more detailed identification of the dispersive bands in the vicinity of the Fermi level; the basic result of such matching is that the experimental bands at low binding energies are the ones predicted by LDA, but renormalized by an average factor of 3 .

Next, we try to understand the origin of the above-described anomalies and deviations from the one-electron picture. For a start, we employ a rather simple model where electrons of the original MFT bands interact with a hypothetical bosonic spectrum [15] according to the Eliashberg formalism. Within this approach, the self-energy, which encapsulates the many-body effects of electronic interactions, is defined by the following formulas, $\Sigma^{\prime}(\omega)=\int_{0}^{\infty} \alpha^{2} F(\Omega) \ln \left|\frac{\omega+\Omega}{\omega-\Omega}\right| d \Omega$, $\Sigma^{\prime \prime}(\omega)=-\pi \int_{0}^{\omega} \alpha^{2} F(\Omega) d \Omega$, where $\Sigma(\omega)=\Sigma^{\prime}(\omega)+i \Sigma^{\prime \prime}(\omega)$ is the self-energy, and $\alpha^{2} F(\Omega)$ is the Eliashberg function.

In Fig. 2 we compare the calculated spectral function with the experimental energy-momentum distribution of the photoemission intensity. The bosonic spectrum $\alpha^{2} F(\Omega)$ was assumed to be of a simplest single-peak shape. In the Supplemental Material [14] we present a convenient analytic form for $\alpha^{2} F(\Omega)$, allowing for an explicit integration of the expressions for $\Sigma^{\prime}$ and $\Sigma^{\prime \prime}$. To make the comparison more transparent, the experimental conditions were chosen in such a way that the photoemission matrix elements highlight one of the bands, while all others are suppressed. The model captures many important features seen in the experimental spectra. First of all, these are the sharp and strongly renormalized dispersions close to the Fermi level. The fast increase of the electronic scattering rate with binding energy at a correct energy scale is reproduced, too-well-defined dispersions vanish below $0.5 \mathrm{eV}$. Finally, the smeared out spectral weight is also distributed around the contours of bare dispersion. This result clearly implies that combining the LDA calculations with the simple treatment of the self-energy satisfactorily reproduces the experimental spectral function of the whole valence band on an energy scale of up to $6 \mathrm{eV}$. Obviously, $\alpha^{2} F(\Omega)$ does not necessarily represent here a particular external bosonic excitation-it could well be just a convenient representation of purely electron-electron interactions, and it remains an open question whether the corresponding bosonic excitations can be singled out.
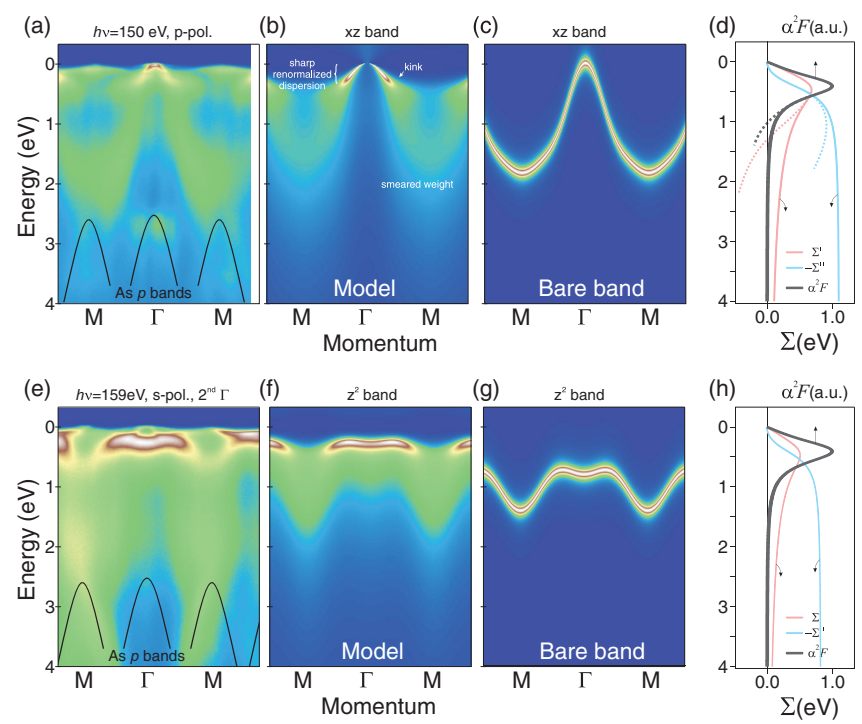

FIG. 2. Model for the spectral function based on the bare dispersion and electronic interaction with a hypothetical bosonic spectrum of single-peak form. Experimental conditions were chosen to highlight one of the bands and suppress the intensity from all others. (a) Experimental data, recorded at a photon energy of $150 \mathrm{eV}$ with vertical light polarization. (b) Spectral function, obtained for the lower-lying $x z / y z$ band and $\lambda=2$. (c) Spectral function for the bare band. (d) Bosonic spectrum $\alpha^{2} F(\omega)$ and self-energy $\Sigma(\omega)$ used in the model. Dashed lines schematically represent the situation where $\Sigma^{\prime}$ changes sign and the value of $\Sigma^{\prime \prime}$ decreases at high $\omega$-this would further improve the agreement between the model and experiment at the bottom of the iron band. (e) Experimental data, recorded at a photon energy of $159 \mathrm{eV}$ with horizontal light polarization in the second Brillouin zone. (f) Spectral function, obtained for the $z^{2}$ band and $\lambda=1.6$. (g) Spectral function for the bare band. (h) Corresponding $\alpha^{2} F(\omega)$ and $\Sigma(\omega)$. 
(a)

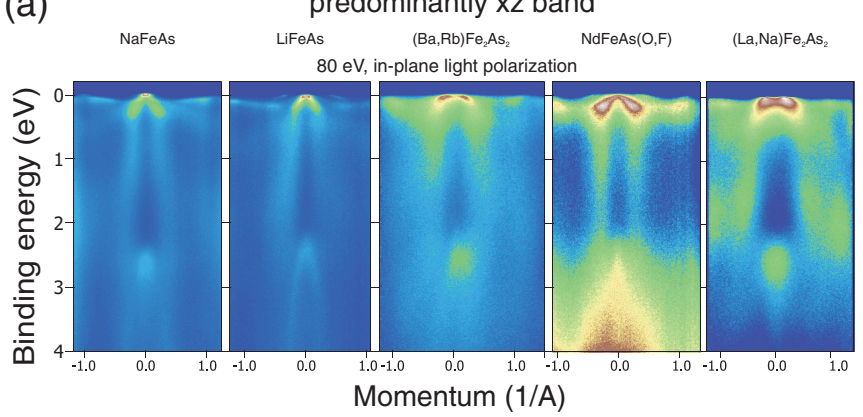

(b)

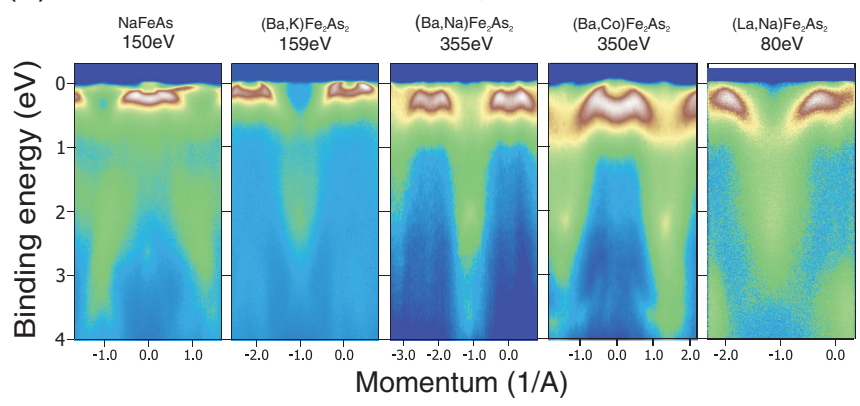

FIG. 3. Experimental data taken from various iron-based superconductors, revealing common signatures of strong electron interactions in the spectral function. (a) Data recorded from $\mathrm{NaFeAs}$, LiFeAs, (Ba, Rb)Fe $\mathrm{Fs}_{2}, \mathrm{NdFeAs}(\mathrm{O}, \mathrm{F})$, and (La,Na)Fe $\mathrm{As}_{2}$. All spectra were taken along the $\Gamma M$ direction, Brillouin zone diagonal, exclusively with the light of $80 \mathrm{eV}$ energy and polarization lying in the $a b$ plane. These experimental conditions highlight the spectral function related to the original $x z$ band. Note the similarities in all important aspects for the spectra of different materials and compare to the corresponding model, (b) and (c). (b) Experimental data recorded from $\mathrm{NaFeAs}$, $(\mathrm{La}, \mathrm{Na}) \mathrm{Fe}_{2} \mathrm{As}_{2}$, and hole- and electron-doped $\mathrm{BaFe}_{2} \mathrm{As}_{2}$ along the $\Gamma M$ direction. Experimental conditions were tuned in order to highlight the spectral weight originating from the original $z^{2}$ band. The presented data reveal signatures of strong electron interactions and a similar spectral shape (fat letter $M$ ) is observed for all studied materials; also see (f) and ( $\mathrm{g}$ ).

In order to illustrate the universality of the high-energy anomaly for a large class of materials, we show ARPES spectra recorded from different iron arsenides of 111, 122, and 1111 crystalline structures with electron, hole, or no doping in Fig. 3. In Fig. 3(a) solely the energy-momentum cuts passing through the $\Gamma$ point, recorded with $80 \mathrm{eV}$ photons of vertical polarization, are presented. At these conditions the most contribution to the photoemission intensity comes from the $x z$ band, while the matrix element for other bands is largely suppressed. The observed intensity distributions are very similar to each other and possess all signatures of strong electron interactions of a high-energy scale, discussed above [also see Figs. 2(a) and 2(b)] - most prominently, the region of intense well-defined dispersion near the Fermi level ends sharply, leaving only a broad spectral weight at higher binding energies. Figure 3(b) shows data recorded with experimental parameters highlighting the $z^{2}$ band. The spectral weight here also exhibits all the features, inherent to the interacting electronic systems. In particular, the intensity in the shape of the letter $\mathrm{M}$ centered at zero momentum is seen in all cases.
One may notice that the experimental spectral function slightly deviates from the model in the region between 1.5 and $2 \mathrm{eV}$ : The contours of the smeared spectral weight distribution are somewhat below the original bare bands and the electronic scattering at the bottoms of these bands is somewhat lower [Figs. 2(a) and 2(b)]. Both these discrepancies can be removed by allowing a decrease of the scattering rate after it passes the maximum, which would cause a simultaneous change of sign of the real part of the self-energy [Fig. 2(d)]. Interestingly, the same effect is observed when one describes the spectra of cuprates, ruthenates, and vanadates using the self-energy formalism [16-18]. Within our model such behavior of the self-energy would necessarily imply that the bosonic spectrum $\alpha^{2} F$ becomes negative, starting from a particular energy. The peculiarities of the experimental spectra, including a high-energy kink, are very reminiscent (both in shape and energy scale) of the features present in the spectral function, calculated for strongly correlated electronic systems [5,19-22]. Together, it means that while the intensity distribution corresponding to the $\mathrm{Fe} 3 d$ band, as it appears in iron pnictides and chalcogenides, can be described by weak-coupling equations surprisingly adequately, at the same time it inherits some signatures peculiar to the strong-coupling approach.

The observation of high-energy anomalies in the experimental spectral function such as a kink in dispersion and a stripe of intensity depletion as well as the possibility to track intensity variations at the contours of original MFT bands allow us to determine the Eliashberg function rather precisely. What could be the physical nature of the introduced effective bosonic spectrum $\alpha^{2} F$ ? A phononic origin can be ruled out, as the typical energies of phononic modes have more than an order of magnitude lower energy [23,24]. One of the obvious candidates would be the spin-fluctuation spectrum, as strong electron coupling to the spin resonance mode below $T_{\mathrm{c}}$ has been detected for many iron-based superconductors [25-27]. However, the spin-fluctuation spectrum has a maximum at about $200 \mathrm{meV}$, and does not extend as high as $500 \mathrm{meV}$ $[28,29]$. Consequently, although both spin fluctuations and phonons certainly make contributions to the band renormalization at lower binding energies, they cannot be a source of the high-energy anomalies and band renormalization on the largest energy scale of the $3 d$ band discussed here. Among other theoretically considered possibilities, the Coulomb interaction in the forms of (i) the well-known on-site repulsion $U$ and (ii) recently proposed to be important in iron-based superconductors Hund's coupling $J$ [30-32] are reasonable candidates for explanations of high-energy anomalies in the electronic spectrum. More theoretical and experimental work is obviously needed to understand the origin and details of the spectral anomalies introduced here.

It is instructive to recall that most of the electronic systems are "normal" - their spectra do not exhibit such strong anomalies and departures from MFT. We show ARPES spectra for a number of renowned materials in Fig. 4(a). The band dispersion can be traced down to a $5 \mathrm{eV}$ binding energy and even deeper, and the agreement with MFT including the energy bandwidth is nearly perfect. A good intuitive quantity for an illustration of the electronic interaction strength is the scattering rate $\Sigma^{\prime \prime}$. A plot of the binding energy dependence $\Sigma^{\prime \prime}(\omega)$ for 

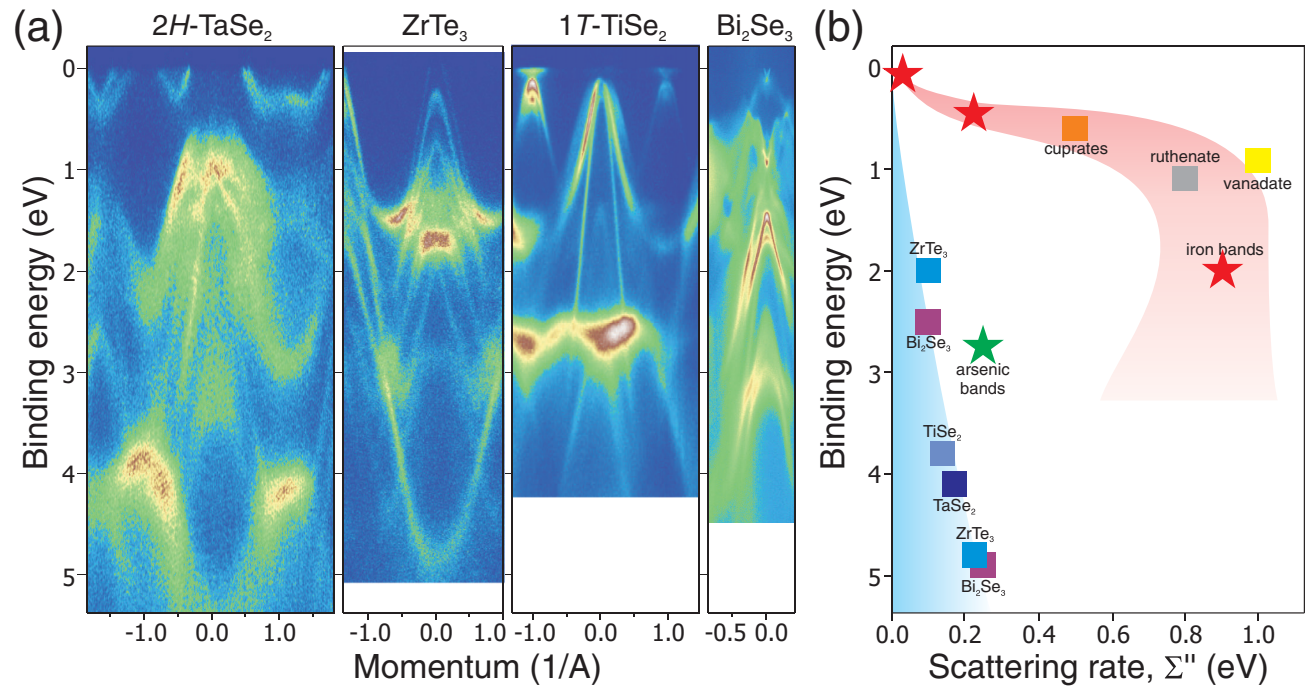

FIG. 4. Dependence of the scattering rate on the binding energy in different compounds. In the materials with relatively weak electronic interactions, such as $\mathrm{TaSe}_{2}, \mathrm{ZrTe}_{3}, \mathrm{TiSe}_{2}$, and $\mathrm{Bi}_{2} \mathrm{Se}_{3}$, the spectra are sharp, and the band dispersion remains well discernible down to $5 \mathrm{eV}$ and further from the Fermi level. In contrast, for the materials referred to as "correlated," or featuring strong electron interactions, such as iron-based, cuprate and ruthenate superconductors, and vanadates, the scattering rate grows very fast, and sharp dispersing features are observed only within several hundreds of meV from the Fermi level. (a) ARPES spectra of $\mathrm{TaSe}_{2}, \mathrm{ZrTe}_{3}, \mathrm{TiSe}_{2}$, and $\mathrm{Bi}_{2} \mathrm{Se}_{3}$. (b) $\mathrm{Scattering}$ rate, determined from the width of energy distribution curves; data for cuprates from Refs. [16,33]; for ruthenates from Ref. [17]; for vanadates from Ref. [18].

several materials is adduced in Fig. 4(b). There is a drastically different behavior of the scattering rates for groups of materials with and without substantial electronic interactions.

Certainly, the most interesting question is the relation of the above-discussed large-scale electronic interaction to the electron pairing. It is a priori clear that the introduced effective bosonic spectrum can hardly be considered as a pairing interaction in a conventional sense. Moreover, if one makes an attempt to estimate $T_{\mathrm{c}}$ with the parameters of the extracted spectrum, $\lambda \approx 2, \omega_{\text {average }} \approx 0.5 \mathrm{eV}$, then with the original BCS formula one arrives at $T_{\mathrm{c}} \approx \omega_{\text {average }} \exp ^{-1 / \lambda}=3600 \mathrm{~K}$, and at $970 \mathrm{~K}$ with McMillan's expression [37] —as expected, one gets values that are way too high. On the other hand, there is a rather clear hint for the importance of such electronic interactions for high-temperature superconductivity: It is present in all studied high- $T_{\mathrm{c}}$ superconductors. Finally, there are a number of materials isostructural to iron arsenides which were synthesized with complete substitution of one or more elements, e.g., $\mathrm{BaCo}_{2} \mathrm{As}_{2}$ [38], $\mathrm{BaNi}_{2} \mathrm{As}_{2}$ [39], and $\mathrm{SrPd}_{2} \mathrm{Ge}_{2}$
[40]. In all of these materials superconductivity is either absent, or $T_{\mathrm{c}}$ is low and superconductivity is believed to be of a conventional phonon origin. Remarkably, the band renormalization peculiar to iron-based superconductors, in all these materials, is not observed. Thus, there is a strong empirical indication for strong electronic interactions on the energy scale of the whole $d$ band to be a necessary requisite for unconventional high-temperature superconductivity.

We thank I. Nekrasov, A. Boris, A. Charnukha, and D. Inosov for helpful discussions, and to R. Hübel, S. Leger, and M. Naumann for technical support. The work was supported under Grants No. BO1912/2-2, No. BE1749/13, and No. WU595/3-1. A.K. appreciates funding from NAS of Ukraine (Project No. 73-02-14). S.W. and I.M. thank the VWfoundation for financial support. I.M. appreciates funding from RSF, Grant No. 164201100 and RFBR (15-03- 99628-a). S.W. thanks the BMBF for support in the frame of the ERA.Net RUS project (Project No. 01DJ12096, FeSuCo).
[1] R. O. Jones and O. Gunnarsson, Rev. Mod. Phys. 61, 689 (1989).

[2] M. C. Payne, M. P. Teter, D. C. Allan, T. A. Arias, and J. D. Joannopoulos, Rev. Mod. Phys. 64, 1045 (1992).

[3] W. W. Schulz, P. B. Allen, and N. Trivedi, Phys. Rev. B 45, 10886 (1992).

[4] E. Dagotto, Rev. Mod. Phys. 66, 763 (1994).

[5] M. Imada, A. Fujimori, and Y. Tokura, Rev. Mod. Phys. 70, 1039 (1998).

[6] G. R. Stewart, Rev. Mod. Phys. 83, 1589 (2011).

[7] J. Paglione and R. L. Greene, Nat. Phys. 6, 645 (2010).

[8] P. Popovich, A. V. Boris, O. V. Dolgov, A. A. Golubov, D. L. Sun, C. T. Lin, R. K. Kremer, and B. Keimer, Phys. Rev. Lett. 105, 027003 (2010).
[9] S. V. Borisenko, V. B. Zabolotnyy, D. V. Evtushinsky, T. K. Kim, I. V. Morozov, A. N. Yaresko, A. A. Kordyuk, G. Behr, A. Vasiliev, R. Follath, and B. Büchner, Phys. Rev. Lett. 105, 067002 (2010).

[10] H. Ding, K. Nakayama, P. Richard, S. Souma, T. Sato, T. Takahashi, M. Neupane, Y.-M. Xu, Z.-H. Pan, and A. V. Fedorov, J. Phys.: Condens. Matter 23, 135701 (2011).

[11] S. T. Cui, S. Y. Zhu, A. F. Wang, S. Kong, S. L. Ju, X. G. Luo, X. H. Chen, G. B. Zhang, and Z. Sun, Phys. Rev. B 86, 155143 (2012).

[12] D. V. Evtushinsky, V. B. Zabolotnyy, T. K. Kim, A. A. Kordyuk, A. N. Yaresko, J. Maletz, S. Aswartham, S. Wurmehl, A. V. Boris, D. L. Sun, C. T. Lin, B. Shen, H. H. Wen, A. Varykhalov, 
R. Follath, B. Büchner, and S. V. Borisenko, Phys. Rev. B 89, 064514 (2014).

[13] A. Charnukha, P. Popovich, Y. Matiks, D. L. Sun, C. T. Lin, A. N. Yaresko, B. Keimer, and A. V. Boris, Nat. Commun. 2, 219 (2011).

[14] See Supplemental Material at http://link.aps.org/supplemental/ 10.1103/PhysRevB.96.060501 for a more detailed identification of the dispersive bands in the vicinity of the Fermi level, derivation of convenient formulas for the self-energy, and for details of the spectral function analysis in a wide range of binding energy.

[15] From the practical side there are good reasons to operate the notion of a hypothetical bosonic spectrum here: (i) Real, and imaginary, parts of the self-energy, introduced by the presented integrals, always appear to be Kramers-Kronig consistentotherwise, one would have to control the consistency separately; (ii) the self-energy, describing the experiment, corresponds to the $\alpha^{2} F$ of a rather simple single-peak shape. From a bit more general perspective, it is not completely surprising that the interaction of a given particle with the rest of the electronic system can be approximated in terms of a scattering on some (broad) bosonic spectrum. Certainly, we do not keep in mind any well-defined excitations, but rather think that it may be possible to single out some (let them be poorly defined) corresponding modes.

[16] W. Meevasana, X. J. Zhou, S. Sahrakorpi, W. S. Lee, W. L. Yang, K. Tanaka, N. Mannella, T. Yoshida, D. H. Lu, Y. L. Chen, R. H. He, H. Lin, S. Komiya, Y. Ando, F. Zhou, W. X. Ti, J. W. Xiong, Z. X. Zhao, T. Sasagawa, T. Kakeshita, K. Fujita, S. Uchida, H. Eisaki, A. Fujimori, Z. Hussain, R. S. Markiewicz, A. Bansil, N. Nagaosa, J. Zaanen, T. P. Devereaux, and Z. X. Shen, Phys. Rev. B 75, 174506 (2007).

[17] H. Iwasawa, Y. Yoshida, I. Hase, K. Shimada, H. Namatame, M. Taniguchi, and Y. Aiura, Phys. Rev. Lett. 109, 066404 (2012).

[18] S. Aizaki, T. Yoshida, K. Yoshimatsu, M. Takizawa, M. Minohara, S. Ideta, A. Fujimori, K. Gupta, P. Mahadevan, K. Horiba, H. Kumigashira, and M. Oshima, Phys. Rev. Lett. 109, 056401 (2012).

[19] A. Georges, G. Kotliar, W. Krauth, and Rozenberg, Rev. Mod. Phys. 68, 13 (1996)

[20] K. Held, Adv. Phys. 56, 829 (2007).

[21] A. Macridin, M. Jarrell, T. Maier, and D. J. Scalapino, Phys. Rev. Lett. 99, 237001 (2007).

[22] K. Byczuk, M. Kollar, K. Held, Y.-F. Yang, I. A. Nekrasov, Th. Pruschke, and D. Vollhardt, Nat. Phys. 3, 168 (2007).

[23] L. Boeri, O. V. Dolgov, and A. A. Golubov, Physica C 469, 628 (2009).

[24] T. Fukuda, A. Q. R. Baron, H. Nakamura, S.-i. Shamoto, M. Ishikado, M. Machida, H. Uchiyama, A. Iyo, H. Kito, J. Mizuki, M. Arai, and H. Eisaki, J. Phys. Soc. Jpn. 80, SB015 (2011).

[25] P. Richard, T. Sato, K. Nakayama, S. Souma, T. Takahashi, Y.-M. Xu, G. F. Chen, J. L. Luo, N. L. Wang, and H. Ding, Phys. Rev. Lett. 102, 047003 (2009).
[26] A. Charnukha, O. V. Dolgov, A. A. Golubov, Y. Matiks, D. L. Sun, C. T. Lin, B. Keimer, and A. V. Boris, Phys. Rev. B 84, 174511 (2011).

[27] A. D. Christianson, E. A. Goremychkin, R. Osborn, S Rosenkranz, M. D. Lumsden, C. D. Malliakas, I. S. Todorov, H. Claus, D. Y. Chung, M. G. Kanatzidis, R. I. Bewley, and T. Guidi, Nature (London) 456, 930 (2008).

[28] P. C. Dai, J. P. Hu, and E. Dagotto, Nat. Phys. 8, 709 (2012).

[29] K.-J. Zhou, Y.-B. Huang, C. Monney, X. Dai, V. N. Strocov, N.-L. Wang, Z.-G. Chen, C. Zhang, P. Dai, L. Patthey, J. van den Brink, H. Ding, and T. Schmitt, Nat. Commun. 4, 1470 (2013).

[30] M. Aichhorn, L. Pourovskii, V. Vildosola, M. Ferrero, O. Parcollet, T. Miyake, A. Georges, and S. Biermann, Phys. Rev. B 80, 085101 (2009).

[31] K. Haule and G. Kotliar, New J. Phys. 11, 025021 (2009).

[32] J. Ferber, K. Foyevtsova, R. Valenti, and H. O. Jeschke, Phys. Rev. B 85, 094505 (2012).

[33] The anomaly known in cuprates as the high-energy kink, or waterfall effect, is strongly influenced by the photoemission matrix elements [34]. Nevertheless, basing on the thorough data analysis [35] and on the $a b$ initio theoretical calculation of the matrix elements, together with the spectral function modeling [36], it has been demonstrated that there are both intrinsic and extrinsic contributions to the spectral anomaly. The situation in cuprates was difficult due to the presence of only one relevant portion of the band structure, and the close vicinity of the enormously intense oxygen bands. In the iron-based superconductors these technical problems are much less acute-the As-derived states are faint, and there are several bands offering the possibility to study the spectral function in full, including the high-energy part.

[34] D. S. Inosov, J. Fink, A. A. Kordyuk, S. V. Borisenko, V. B. Zabolotnyy, R. Schuster, M. Knupfer, B. Büchner, R. Follath, H. A. Dürr, W. Eberhardt, V. Hinkov, B. Keimer, and H. Berger, Phys. Rev. Lett. 99, 237002 (2007); D. S. Inosov, R. Schuster, A. A. Kordyuk, J. Fink, S. V. Borisenko, V. B. Zabolotnyy, D. V. Evtushinsky, M. Knupfer, B. Büchner, R. Follath, and H. Berger, Phys. Rev. B 77, 212504 (2008).

[35] W. Meevasana, F. Baumberger, K. Tanaka, F. Schmitt, W. R. Dunkel, D. H. Lu, S.-K. Mo, H. Eisaki, and Z.-X. Shen, Phys. Rev. B 77, 104506 (2008).

[36] S. Basak, T. Das, H. Lin, J. Nieminen, M. Lindroos, R. S Markiewicz, and A. Bansil, Phys. Rev. B 80, 214520 (2009).

[37] J. P. Carbotte, Rev. Mod. Phys. 62, 1027 (1990).

[38] N. Xu, P. Richard, A. van Roekeghem, P. Zhang, H. Miao, W.-L. Zhang, T. Qian, M. Ferrero, A. S. Sefat, S. Biermann, and H. Ding, Phys. Rev. X 3, 011006 (2013).

[39] B. Zhou, M. Xu, Y. Zhang, G. Xu, C. He, L. X. Yang, F. Chen, B. P. Xie, X.-Y. Cui, M. Arita, K. Shimada, H. Namatame, M. Taniguchi, X. Dai, and D. L. Feng, Phys. Rev. B 83, 035110 (2011).

[40] T. K. Kim, A. N. Yaresko, V. B. Zabolotnyy, A. A. Kordyuk, D. V. Evtushinsky, N. H. Sung, B. K. Cho, T. Samuely, P. Szabó, J. G. Rodrigo, J. T. Park, D. S. Inosov, P. Samuely, B. Büchner, and S. V. Borisenko, Phys. Rev. B 85, 014520 (2012). 